\title{
BMJ Open A cluster randomised controlled trial of advice, exercise or multifactorial assessment to prevent falls and fractures in community-dwelling older adults: protocol for the prevention of falls injury trial (PreFIT)
}

\author{
Julie Bruce, ${ }^{1}$ Ranjit Lall, ${ }^{1}$ Emma J Withers, ${ }^{1}$ Susanne Finnegan, ${ }^{1}$ \\ Martin Underwood, ${ }^{1}$ Claire Hulme, ${ }^{2}$ Ray Sheridan, ${ }^{3}$ Dawn A Skelton, ${ }^{4}$ \\ Finbarr Martin, ${ }^{5}$ Sarah E Lamb, ${ }^{1,6}$ on behalf of PreFIT Study Group
}

To cite: Bruce J, Lall R, Withers EJ, et al. A cluster randomised controlled trial of advice, exercise or multifactorial assessment to prevent falls and fractures in community-dwelling older adults: protocol for the prevention of falls injury trial (PreFIT). BMJ Open 2016;6: e009362. doi:10.1136/ bmjopen-2015-009362

- Prepublication history and additional material is available. To view please visit the journal (http://dx.doi.org/ 10.1136/bmjopen-2015009362).

Received 10 July 2015 Revised 1 September 2015 Accepted 10 September 2015

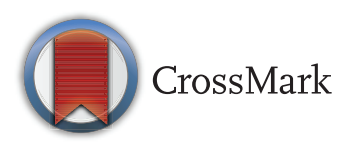

For numbered affiliations see end of article.

Correspondence to Professor Sarah E Lamb; sarah.lamb@ndorms.ox.ac.uk

\section{ABSTRACT}

Introduction: Falls are the leading cause of accidentrelated mortality in older adults. Injurious falls are associated with functional decline, disability, healthcare utilisation and significant National Health Service (NHS)-related costs. The evidence base for multifactorial or exercise interventions reducing fractures in the general population is weak. This protocol describes a large-scale UK trial investigating the clinical and cost-effectiveness of alternative falls prevention interventions targeted at community dwelling older adults.

Methods and analysis: A three-arm, pragmatic, cluster randomised controlled trial, conducted within primary care in England, UK. Sixty-three general practices will be randomised to deliver one of three falls prevention interventions: (1) advice only; (2) advice with exercise; or (3) advice with multifactorial falls prevention (MFFP). We aim to recruit over 9000 community-dwelling adults aged 70 and above. Practices randomised to deliver advice will mail out advice booklets. Practices randomised to deliver 'active' interventions, either exercise or MFFP, send all trial participants the advice booklet and a screening survey to identify participants with a history of falling or balance problems. Onward referral to 'active' intervention will be based on falls risk determined from balance screen. The primary outcome is peripheral fracture; secondary outcomes include number with at least one fracture, falls, mortality, quality of life and health service resource use at 18 months, captured using self-report and routine healthcare activity data. Ethics and dissemination: The study protocol has approval from the National Research Ethics Service (REC reference 10/H0401/36; Protocol V.3.1, 21/May/ 2013). User groups and patient representatives were consulted to inform trial design. Results will be reported at conferences and in peer-reviewed publications. A patient-friendly summary of trial findings will be published on the prevention of falls injury trial (PreFIT) website. This protocol adheres to the recommended SPIRIT Checklist. Amendments will be reported to relevant regulatory parties.

Trial registration number: ISRCTN 71002650; Preresults.

\section{INTRODUCTION}

Falls and fall-related fractures are a major public health burden for individuals and society. Falls are associated with loss of independence, functional decline and are a contributing reason for subsequent admission to a long-term care. ${ }^{1}$ The majority of falls result in minor injury and pain, but are sometimes coupled with a fear of falling and loss of confidence in mobility. Falls, however, can also lead to serious injury, with fracture and hospitalisation occurring in 5\% of community dwelling older adults with a history of falling. ${ }^{2}$ The financial burden associated with injurious falls is staggering: direct healthcare and associated social care costs in the UK have been estimated at $£ 2$ billion per annum, mostly associated with hip fracture. ${ }^{3}$ Costs arise from hospital admission and clinical care associated with treatment of injurious falls and fractures. Mortality is high in people who sustain fracture. For hip fracture, $10 \%$ die within 30 days and one-third die within 12 months. ${ }^{4}$ Demographic change means that injurious falls in older people may become increasingly common.

Over the past 30 years, much effort has been directed towards the implementation and evaluation of fall prevention strategies. Falls have a multifactorial aetiology, with 
many putative risk factors identified. ${ }^{5}$ The major risk factors are diverse, and potentially modifiable, including impairments of gait and balance, visual impairments, syncope and cardiac rhythm abnormalities, polypharmacy and certain classes of medication, comorbidity, foot disorders and environmental hazards. Early clinical trials of assessment and treatment of multiple risk factors, termed multifactorial falls strategies, were very promising, suggesting beneficial effect in falls reduction when compared to usual care. These early trials provided the foundation for the mandatory establishment of secondary prevention in the UK, through the introduction of falls services to undertake multifactorial falls prevention (MFFP) interventions on people with a history of falling. ${ }^{6}$ These trials did not, however, systematically examine the effect of interventions on injurious falls. To justify widespread introduction of services for the primary prevention of falls, evidence is needed that these interventions reduce injurious falls. Multifactorial risk assessment, followed by targeted treatment of individual risk factors, is currently the mainstay for falls prevention in the UK, supported by clinical organisations (American Geriatric Society/British Geriatric Society) and the UK National Institute for Health and Clinical Excellence. ${ }^{7}$ Falls services have been widely introduced throughout the UK National Health Service (NHS), although structure and process evaluation revealed considerable variability in service design, models of delivery and professional skill mix. ${ }^{8} 9$

Numerous trials have been conducted to investigate the efficacy of falls prevention initiatives; a systematic review included 159 trials of different falls prevention strategies, many of which were complex interventions. ${ }^{10}$ Certain exercise programmes, in particular, those focusing on individualised and progressive gait, strength and balance retraining, have been shown to reduce rate of falls by approximately $25 \% .{ }^{10}$ Challenges include exercise adherence, however, strength and balance interventions may be a cost-effective strategy. Worryingly, several systematic reviews have found mixed evidence for multifactorial fall risk assessment and intervention on outcomes of rate of falls and fall-related injuries. Gates $e t a l^{11}$ suggested that multifactorial interventions may reduce the rate of falls without affecting the number of fallers (falls risk), a finding confirmed in an updated Cochrane review. ${ }^{10}$

These systematic reviews identified methodological deficiencies in existing trials, with many studies being underpowered and lacking robust data on important outcomes, including quality of life, fracture, costs of intervention and cost-effectiveness. Of those reviewed, only 11 of 40 published trials of multifactorial interventions included fracture outcomes, despite serious injury causing the greatest burden to patients and contributing to resource use. ${ }^{10}$ No trials have been sufficiently pragmatic to estimate the impact of fall screening and intervention at a population level.

Given the widespread introduction of falls services in the UK and current recommendations for MFFP in certain populations, adequately powered studies are urgently required to investigate the effectiveness of such initiatives on clinical and patient-reported outcomes. This cluster randomised controlled trial is designed to compare the effectiveness of alternative strategies to prevent falls and fractures in community-dwelling older adults.

\section{METHODS AND ANALYSIS \\ Aim}

The overall aim is to determine the comparative effectiveness and cost-effectiveness of three primary care fall prevention interventions: advice alone, advice supplemented with either exercise or MFFP, in adults aged 70 years and above, on outcomes of fracture, falls and quality of life. Specific trial objectives are to: (A) estimate the effectiveness of intervention in communitydwelling people of different age, frailty status, gender and falls history; (B) measure the uptake of and retention to exercise and MFFP (active interventions) and the impact these have on effectiveness; and $(\mathrm{C})$ to assess the relative costs of each intervention and to determine the most cost-effective approach.

\section{Trial design and setting}

Core trial information is presented in table 1 (WHO Trial Registration Data Set). This study is a three-arm pragmatic cluster-randomised controlled trial with parallel economic analysis. The trial design framework is superiority rather than equivalence or exploratory. The trial is currently running in primary care; a minimum of 9000 participants will be recruited from 63 general practices across England. Recruitment is underway in different English regions selected to ensure our population is geographically representative and includes extremes of latitude. We are recruiting in the South-West (Devon), Central (West Midlands, Cambridgeshire) and Northern England (Newcastle). Urban and rural practices will be recruited with support provided by existing Primary Care Research Networks (PCRN) and Comprehensive Local Research Networks (CLRN). A pilot study was undertaken in the Devon Region to assess operational futility, namely to assess the feasibility of setting up recruitment, interventions and referral processes. The Trial Steering Committee (TSC) and funder reviewed pilot study findings before the main trial was launched.

\section{Eligibility criteria—cluster level}

Practice eligibility: General practices are eligible to participate if they are located in an English region with infrastructure to support the trial. Additional eligibility criteria include agreement to adhere to a predetermined active treatment pathway (exercise or MFFP) and technical capacity to undertake electronic searching to identify a suitable population base for sample selection. Modest reimbursement is provided to cover practice time and postage. 
Table 1 WHO Trial Registration Data Set

Data category
Primary registry and trial
identifying number
Date of registration in primary
registry
Secondary identifying numbers
Source of monetary or material
support
Primary sponsor
Secondary sponsor
Contact for public queries
Contact for scientific queries
Public title
Scientific title
Countries of recruitment
Health condition or problem
studied
Interventions

Information

ISRCTN 71002650: Prevention of fall injury trial (PreFIT)

$13 / 04 / 2010$

Health Technology Assessment (HTA)

National Institute for Health Research (NIHR) HTA

University of Warwick

Not applicable

prefit@warwick.ac.uk

Dr Julie Bruce, Warwick Clinical Trials Unit, University of Warwick

PreFIT

PreFIT a parallel group cluster randomised controlled trial and economic evaluation

UK

Falls and fractures in older adults

Advice only: Age UK leaflet

Comparator 1: Exercise: PreFIT-Otago Home Exercise Programme

Comparator 2: Multifactorial falls prevention assessment (MFFP)

Key inclusion and exclusion

Age: 70 years or over, no upper age restriction

Sex: Male or female

Inclusion: Any comorbidity, resident in the community or sheltered housing

Exclusion: Those resident in long-term residential or nursing care, those with terminal

illness or expected shortened life span defined as less than 6 months, as determined by

general practitioner. No restrictions by cognitive functioning, comorbidity or falls history

apply

Study type

Interventional

Allocation: randomised; cluster assignment

Primary purpose: prevention, setting primary care

Phase III

October 2011

Date of first enrolment

Over 9000

Recruiting

Recruitment status

Peripheral fracture

Primary outcome
Key secondary outcomes

Falls, health-related quality of life, mortality, healthcare resource use

\section{Eligibility criteria-participant level}

Participant eligibility: Community-dwelling older adults, defined as aged 70 years or above, resident in the community or in sheltered housing. Exclusions include those in long-term residential or nursing care and those with terminal illness or expected shortened lifespan, defined as less than 6 months, as determined by the general practitioner (GP). No specific restrictions or exclusions by sex, cognitive functioning, comorbidity or falls history apply.

\section{Participant recruitment and consent}

We seek to recruit 150 participants (range 129-179) from each general practice (figure 1). Practices search their electronic database to identify a random sample of 400 older adults aged 70 years and above. After removal of exclusions, practices then mail an invitation pack which contains a participant information sheet, baseline questionnaire and consent form. With an estimated enrolment rate of $35 \%-40 \%$ this yields $140-160$ recruited participants per practice (figure 2). Informed consent is sought from each participant for access to medical records and routine primary and secondary care data held by the UK NHS Health and Social Care Information Centre. Patient-specific data are only collected on those providing consent.

\section{Allocation sequence generation and randomisation}

The unit of cluster randomisation is the general practice (figure 2). Once 150 participants are recruited from three GP practices, practices are then randomised in a block of three to ensure that concealment of the allocation sequence is maintained. Randomisation is based on a computer-generated randomisation algorithm held and controlled centrally within the trials unit by an independent programmer. Once informed of practice allocation, trial administrators then implement screening procedures and research staff plan intervention delivery with local site investigators. 


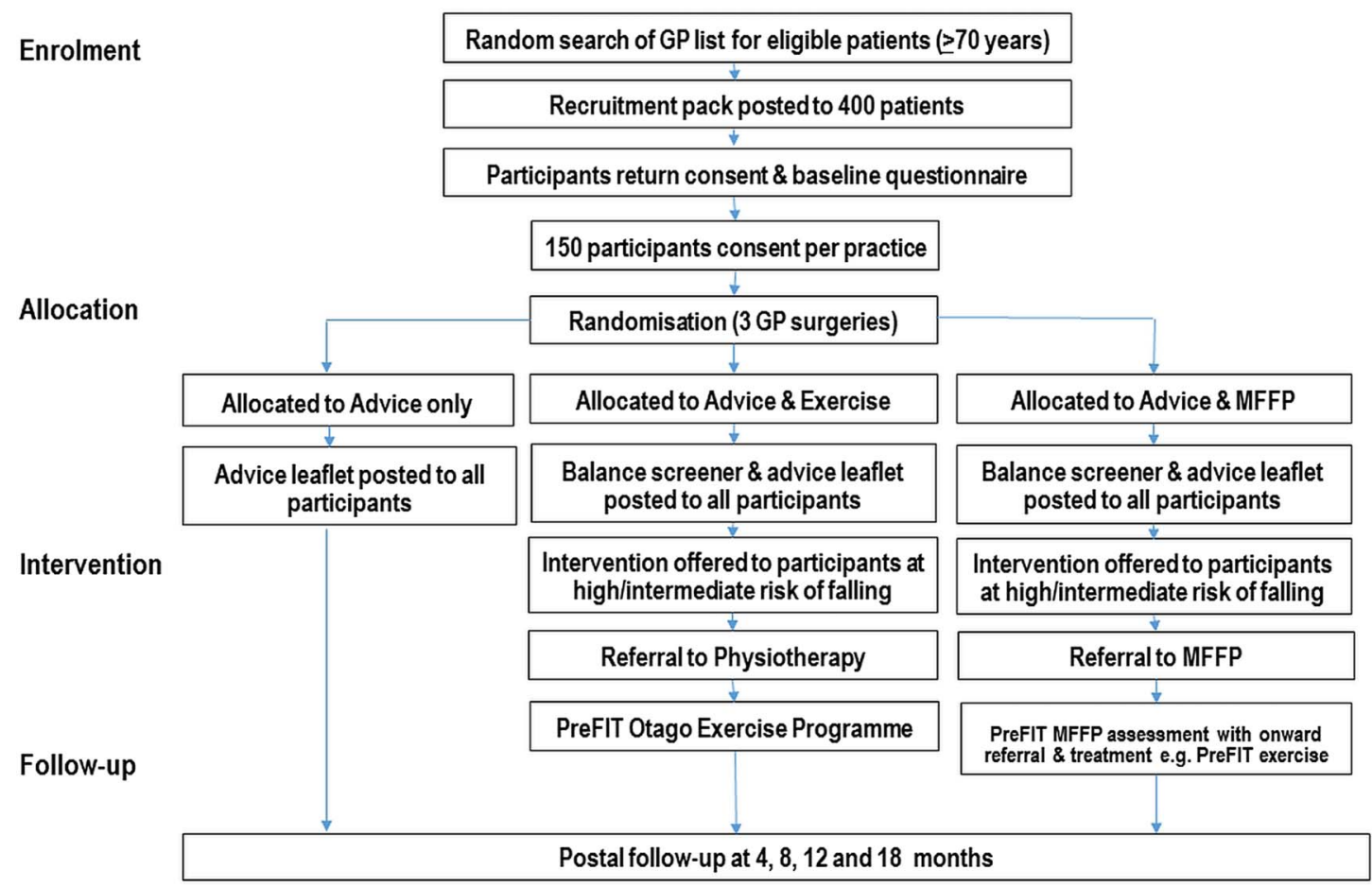

Figure 1 Flow diagram of cluster. MFFP, multifactorial falls prevention; PreFIT, prevention of falls injury trial.

\section{Blinding}

The Consolidating Standards for Reporting Clinical Trials (CONSORT) Statement 2010 and subsequent update extension to cluster randomised trials recommend transparent reporting of blinding status in relation to participants, data analysts, outcome assessors and intervention. ${ }^{12}$ At the invitation stage, patients are consented for data collection and follow-up; they are informed that their GP may contact them with another short questionnaire asking about balance and function, which will be used to determine further advice. At this stage, participants are blinded to treatment options. At the referral to treatment stage, participants are also blinded to other alternate treatment options. Owing to the nature of the prevention of falls injury trial (PreFIT) 'active' interventions, it is not possible to blind therapists or services delivering exercise or MFFP. Blinding of data analysts and outcome assessors is fully achieved. Senior investigators are blinded to treatment allocation for the duration of the conduct of the trial. The statistical analysis plan was developed by the study team and approved by external monitoring committees. Data cleaning and analysis of the primary outcome will be conducted by a trial statistician unaware of treatment allocation. Data entry and processes to ensure data quality will be undertaken by data entry clerks unaware of treatment allocation.

\section{Interventions}

Advice

All trial participants, across all three intervention arms, receive the 'Staying Steady' booklet by post. This 29-page booklet, produced and published by Age UK, contains clear information about improving strength and balance, information about eyesight, hearing, managing medications, the home environment and dealing with advice on fear of falling. Contact numbers and websites for different national help organisations are provided. This colourful information booklet was selected for use because of the positive emphasis on remaining steady and active rather than focus on falls per se. Practices allocated to the 'Advice only' arm deliver no further planned intervention and are not actively screened for balance problems.

\section{Screening and referral to active intervention}

A primary care screening approach is used whereby decision about onward referral of participants to exercise or MFFP is determined from a 1-page short selfcomplete balance and falls screening survey. Balance screening surveys are mailed from and returned to general practices. The balance screen includes questions on falls history and current balance, based on previous research predicting thresholds for falls. ${ }^{13}$ Participants reporting balance problems whilst walking, dressing or with daily activities and those reporting one or more fall in the previous 12 months are categorised as being at intermediate or high risk of falling. These participants are then offered the opportunity to attend for further assessment and treatment, either exercise or MFFP, depending on cluster allocation.

\section{Exercise arm}

The PreFIT exercise intervention is based on the Otago Home Exercise Programme (OEP), which targets lower limb strength, balance retraining and walking. ${ }^{14}{ }^{15}$ 


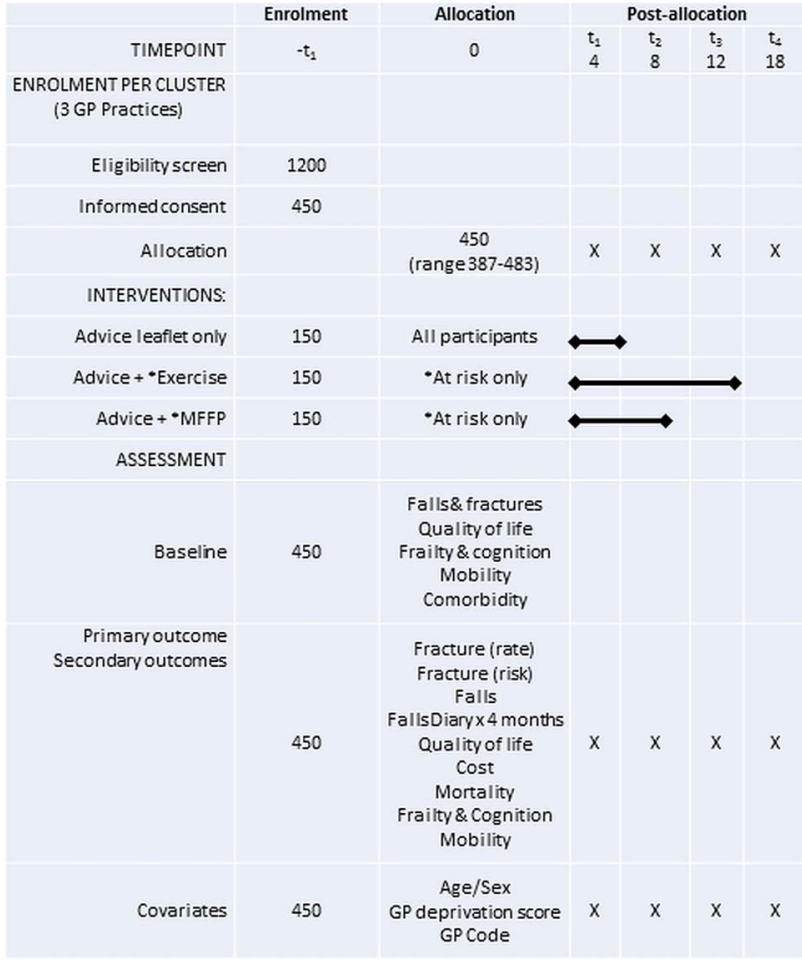

Figure 2 Schedule of enrolment and interventions per cluster (SPIRIT Template). *Active interventions of exercise and MFFP offered to subgroups of participants at intermediate or high risk of falling. Low risk participants in active arms receive advice leaflet only.

Participants eligible for the exercise intervention are invited to attend for baseline assessment, comprising a chair-stand test and four-test balance scale. The OEP is an individually prescribed, progressive exercise programme which can be administered either at home or via group classes by a PreFIT-trained physiotherapist or exercise instructor. The PreFIT-OEP contains five warm-up exercises, five strength exercises with/without ankle cuff weights, and 12 balance retraining exercises. Optimal duration of the PreFIT OEP is 6 months, with a recommended minimum of three face-to-face contacts with telephone support by a trained therapist. Therapists have the flexibility to deliver the intervention in group or individual sessions, depending on their local service configuration. Exercise adherence will be reported as number of participants who fully or partially complete the exercise programme.

\section{MFFP arm, with or without exercise}

The PreFIT MFFP is based on an existing evidencebased MFFP intervention, ${ }^{16}$ with modifications to align with current UK recommendations for falls risk assessment and treatment pathways. ${ }^{6}{ }^{17}$ The following risk factors are assessed on all patients referred for MFFP: comprehensive falls history interview; identification of red flags; gait and balance assessment; consideration of fear of falling; postural hypotension and arrhythmia screen; medication screening and GP-led review of psychotropic and culprit drugs; Snellen visual acuity test; feet and footwear assessment and home environment screening interview. The falls assessment is conducted by a trained healthcare practitioner or falls team, followed by recommendations or further onward referral to another service where indicated. The assessment consists of examinations performed within the general practice, the home, community or general hospital by a practice nurse or equivalent registered healthcare professional, or by a community or hospital-based falls team. The location of the falls assessment will vary due to the pragmatic trial design. Standardised treatment recommendations are linked to each of the risk factors. For example, participants receiving MFFP who have impaired gait and balance or fear of falling are eligible for referral to PreFIT OEP intervention. This is consistent with all models of MFFP delivery, is purposeful and does not represent contamination between the intervention arms.

Detailed description of PreFIT active interventions including the selection of MFFP risk factors, development of exercise and MFFP manuals, testing of interventions and processes for training of healthcare professionals and quality assurance will be reported in a separately, as per recommended guidance for trial intervention description and replication. ${ }^{18}$

\section{Cotreatments}

Trial participants are free to seek management of falls and any other related or unrelated medical conditions during the course of the trial. We place no restriction on other agencies contacting participants about fall prevention strategies. We record all health service resource use and these will be reported as a trial outcome. At trial closure, participants will continue with usual healthcare, no further ancillary care is provided beyond that immediately required for the proper and safe conduct of the trial.

\section{Intervention fidelity checks and process evaluation}

We will report intervention fidelity, process and compliance using a mixture of structured treatment records and observation during quality assurance visits. The trial treatment record includes details on dose and mode of delivery of the major elements of the MFFP and exercise interventions, as well as the grade and type of staff involved with the delivery.

\section{Outcomes}

\section{Primary outcome}

The primary outcome is peripheral fracture, expressed as the fracture rate per person per months of observation over 18 months after randomisation of the general practice. Peripheral fracture, defined according to an internationally agreed definition, ${ }^{19}$ includes any fracture in the appendicular skeleton, thus limbs, limb girdles, cranial and facial bones. Compression fractures within the vertebral column will be excluded from the primary outcome. Fracture data will be obtained from three 
Table 2 Overview of data collection and outcome measures

\begin{tabular}{|c|c|c|}
\hline Variable & Data source and instrument & Time point (months) \\
\hline $\begin{array}{l}\text { Primary outcome } \\
\text { Peripheral fracture }\end{array}$ & $\begin{array}{l}\text { HES inpatient (ICD-10, OPCS codes) } \\
\text { HES A\&E } \\
\text { GP records (Read Codes CTV2 \& 3) } \\
\text { Self-report CRF }\end{array}$ & $0-18$ months \\
\hline Secondary outcomes & Retrospective & \\
\hline Falls & $\begin{array}{l}\text { CRF-Falls in previous } 12 \text { months } \\
\text { CRF-Falls in previous } 4 \text { months } \\
\text { Prospective }\end{array}$ & $4,8,12,18$ \\
\hline & Monthly falls diary & 0-12 months \\
\hline Health-related quality of life & $\begin{array}{l}\text { CRF-Short-Form } 12 \text { (V.2) } \\
\text { CRF-EuroQol EQ-5D }\end{array}$ & $\begin{array}{l}0,4,8,12,18 \\
0,4,8,12,18\end{array}$ \\
\hline Mortality & GP records/HES & $0-18$ months \\
\hline Covariates & & \\
\hline $\begin{array}{l}\text { Ethnicity/marital status/living arrangements/ } \\
\text { education (years) }\end{array}$ & CRF & 0 \\
\hline Height/weight (BMI) & CRF & 0 \\
\hline Mobility & CRF & $0,4,8,12,18$ \\
\hline Frailty & CRF-Strawbridge ${ }^{21}$ & 0,18 \\
\hline Clock drawing test & CRF-Shua-Haim 22 & 0 \\
\hline Healthcare resource use & $\begin{array}{l}\text { CRF-Primary care consultations } \\
\text { CRF-Secondary care attendances } \\
\text { (NHS and private) }\end{array}$ & $4,8,12,18$ \\
\hline
\end{tabular}

sources: Hospital Episode Statistics (HES), self-report from patients and a targeted search of GP records. The Health and Social Care Information Centre (HSCIC) is the national body for mandatory data collection from hospitals in England; HES data on the length, type and reasons for all NHS inpatient hospital admissions, accident and emergency (A\&E) and outpatient clinic attendances will be obtained for trial participants. These data sets include private patients treated in NHS hospitals. Searches of these HES data sets will be undertaken to identify relevant International Classification of Diseases V.10 codes (ICD-10) diagnosis and treatment codes (table 2). Fracture data will also be obtained from selfreport from follow-up questionnaires at 4, 8, 12 and 18 months and a targeted search of general practice records (using READ Codes and free-text). Self-reported fracture is captured using questions about falls-related broken bones collected at baseline and follow-up. Participants reporting any broken bone are contacted by post and telephone interview to collate additional information on fracture site, date and hospital treatment. Although no single data set can be considered as $100 \%$ accurate and timely, any ICD-10 fracture diagnosis reported on HES in-patient data will be accepted as a confirmed event (figure 3). A triangulation approach will be used: any HES A\&E fracture code, any selfreported fracture or any fracture from GP Read Code screening will be investigated further by undertaking a full search within GP-held patient records for the relevant time period (eg, X-ray reports, hospital discharge or clinic letters). Data sources will be cross-checked against other sources. A data validation protocol, approved by external trial committees, will be used to determine suspected and confirmed fracture events. A clinical review panel, blinded to intervention allocation, will review all data sources for any suspected/unconfirmed fracture. In the event that HES data are not available, the trial will recourse to a protocol based on self-report and GP records.

\section{Secondary outcomes and baseline data}

Secondary outcomes include number of patients with at least one fracture, falls, mortality, health-related quality of life and resource use. These outcomes will be captured also by postal questionnaires administered at baseline, 4, 8, 12 and 18 months (table 2). Outcome measures will be collected at baseline, with additional information on demographic characteristics, selfreported comorbidity and a brief frailty screen. Pilot data collection instruments and balance screeners were developed with input and feedback from patient representatives within the target age group; instruments were revised accordingly.

\section{Falls data collection}

Falls definition: we will use an internationally agreed definition of a fall as being an event in which the participant comes to rest on the ground, floor or other lower level. ${ }^{19}{ }^{20}$ This item is phrased as: "in the last 12 months, have you had any fall including a slip or trip; following which you have come to rest on the ground, floor or lower level?". The reference timeframe is altered according to timing of 


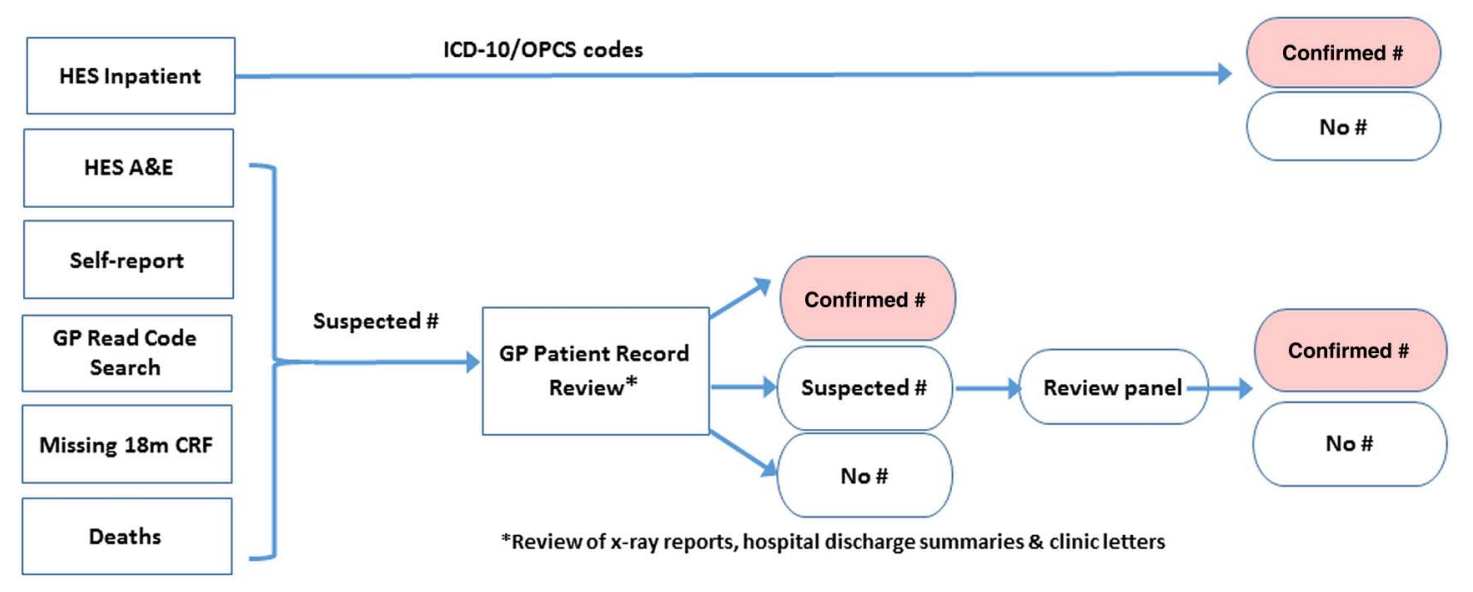

Figure 3 PreFIT Fracture Algorithm. A\&E, accident \& emergency; CRF, case record form; HES, Hospital Episode Statistics; ICD, International Classification of Diseases; PreFIT, prevention of falls injury trial.

follow-up such that the reporting period covers the time since the last follow-up.

Falls recording: Two approaches will be used to capture falls: retrospective self-report (for a reporting period of the preceding months) for each individual over the entire follow-up period and prospective monthly falls diaries. All participants complete a prospective falls diary on a daily basis for a 4-month period during the first year. The collection of falls data by diary is a substantial effort for participants and is resource intensive. Because the analysis of falls data requires substantially fewer participants than the fracture outcome, we designed a strategy of identifying a random subsample of participants to provide diary data in each 4-month period of follow-up, up to 12 months. A computer-generated programme randomly allocates participants to their prospective data collection time point, thus 0-4 months, 4-8 months or 8-12 months from randomisation. Random sampling is without replacement. Prepaid postal diaries are completed each day then returned using to the trial office at the end of each month. Diary data will be used in two ways. We will examine biases in the retrospective fall data reporting, using the fall diary as the gold standard to determine whether the retrospective report can be considered a robust data source. In addition, we will model falls data using the diary reports for the first year of follow-up.

Mobility/ADLs: questions on difficulties balancing on a level surface, ability to walk outside of the house, and average time spent walking will be included at baseline and each subsequent follow-up.

Frailty: A 16-item frailty questionnaire is included at baseline and 18 months. ${ }^{21}$ This measure is weighted towards sensory assessment.

Cognitive ability: A clock-drawing test is included at baseline as a cognitive screening. We will use a sixpoint scoring system, based on the visual-spatial aspects and correct denotation of time by the hand of the clock. $^{22}$

\section{Health-related quality of life}

Health-related quality of life is captured using the Short-Form 12 and EuroQol 5D. The SF-12 provides a measure of physical function, engagement in usual activities and mental functioning. ${ }^{23}$ The EQ5D is a standardised measure of self-reported health-related quality of life which includes five domains: mobility, self-care, usual activities, pain/discomfort and anxiety/ depression. $^{24}$

\section{Resource use}

Health resource use will be captured from routine data sources (including HES) and from participant selfreport questionnaire. Unit cost data will be obtained from national databases such as the British National Formulary and Personal Social Services Research Unit (PSSRU) Costs of Health and Social Care.

\section{Medication use}

We will obtain drug data on trial participants from general practices for two time periods, before and after randomisation. Drug classes of interest will include psychotropics and bone protection drugs (bisphosphates and mineral supplementation). Psychotropic medications are known to increase risk of falling; this drug classification includes: antidepressants; psychotropics; sedatives; hypnotics; anxiolytics and antimanic medications (as per British National Formulary (BNF) classes 4.1.1-4.3.1). Bisphosphonates, often prescribed with calcium and vitamin D supplementation, help slow bone loss and may decrease the risk of fractures (BNF class 6.6.2).

Drug data collection will be undertaken on a random sample of general practices, stratified by intervention arm; drug data will be extracted on all trial participants within the selected practice. Two periods for drug prevalence will be used: (A) baseline: a three calendar month period prior to month of randomisation, and (B) follow-up: a 3-month calendar period at 9-12 months postrandomisation. Drug data will be analysed as 
number of participants prescribed a psychotropic or bone protection drugs and change in number of prescribed psychotropics/bone protection drugs by intervention arm.

\section{Sample size}

The study has been powered using the proportion of patients with at least one fracture, although the primary analysis will be based on a comparison of incidence rate ratios. This is because the literature offers little information to inform the selection of key parameters needed to estimate the incidence rate ratio including an accurate estimate of the person time denominator and the coefficient of variation.

In the UK, the annual fracture incidence per 100 men aged 55-74 years is 2.6 (95\% CI 1.9 to 3.3); this rises to 7.6 fractures (95\% CI 4.0 to 11.3 ) per 100 women aged over 55 years. ${ }^{25}$ Adjusting for sex differences, the general estimated annual population-based fracture rate is $6 / 100$ people $(6 \%)$ aged over 70 years, ${ }^{26}$ and this is the estimate we have selected for the control arm of the trial. This estimate does not account for repeated within-individual fractures and hence is a conservative estimate of the population-based fracture rate.

To have $80 \%$ power to detect a statistically $(\mathrm{p}<0.05)$ and clinically relevant, $2 \%$ difference in fracture rate for the main comparisons (advice vs MFFP; advice vs exercise), from $6 \%$ to $4 \%$ (relative risk reduction, 0.67), requires 1900 participants per arm, or 5700 participants overall. Incorporating a design effect to adjust for varying degrees of modest clustering (ICC set as 0.003), inflates the sample size estimate to 7800 , or 2600 per arm. Allowing for $15 \%$ loss to follow-up, yields a target sample size of 9000 participants. To recruit 9000 at 150 participants per general practice (range 129-179), requires 60 general practices to yield a participant sample range $7740-10740$.

\section{Data analysis}

The primary analysis will be by intention to treat. Initial comparisons will be drawn between advice and the two active intervention arms, and then if appropriate between exercise and MFFP. Initial data presentation will focus on simple descriptive, graphical and tabular summaries as per CONSORT reporting standards for cluster RCTs, including response to screening, uptake of interventions and ongoing retention of participants by treatment arm. ${ }^{12}$ Response rate to postal balance screeners will be assessed for screening yield. Random effect Poisson model/negative Binomial model will be used to assess the primary outcome and will take account of the 18-month outcome data. The data will be analysed using these models and the fit of the models will be assessed. The model which provides the best fit will be used to report the final results, taking account of important predictors such as deprivation score for GP practice, age, gender and general practice (random effect). Peripheral fracture data will be reported using incidence rate (IRR) and their $95 \%$ CI. Likewise, falls data will be analysed using Poisson or negative Binominal models. We will undertake analysis to determine the validity of retrospective falls reporting collected in the follow-up questionnaires, against the prospectively collected falls diary data. We will report and analyse falls data from both data sources, selecting cross-sectional comparisons for the randomly sampled, prospectively collected diary data during each of the discrete time intervals of follow-up.

Time to first fracture will be analysed using survival analysis methods, accounting for the cluster component. Similar analyses, as used for the fracture data, will be computed for the falls data. For other secondary analyses, linear random effect models will be fitted if the outcomes are continuous and logistic random effects models will be fitted if the outcomes are binary. In the case of no clustering effect, appropriate linear or logistic models will be used.

Sensitivity analysis will be carried out using imputation on the primary outcome variable. Multiple imputation, using the ICE (imputation by chain equations) will be used to generate the imputation data set. The effect of the non-compliance in the study will be explored using complier average causal effect (CACE) or per protocol. Modelling of CACE may be challenging because of data distribution. Compliance and adherence will be fully described outlining the development, content and delivery of the exercise and MFFP interventions. Briefly, adherence will be reported as number of sessions attended; 'engaged with intervention' will include those participants referred for active treatment who attended at least one treatment session. Further detailed analyses on adherence to exercise intervention (number of exercise sessions attended with healthcare professional, number of participants who completed some or all of the prescribed exercise programme) will be undertaken. The data monitoring committee will approve the final version of the data analysis plan.

\section{Planned subgroup analyses}

We have selected the following a priori subgroup criteria based on the published literature. ${ }^{27} 28$ We will explore effectiveness by age, sex, falls history, cognitive impairment and frailty. Although we are unlikely to encounter large numbers of people living in the community with severe cognitive impairment, the ability to engage in falls prevention strategies may be affected by mild to moderate levels of cognitive impairment. Subgroup effects will be tested through formal interaction tests. ${ }^{29}$

\section{Data security}

Participant data are being stored on a secure database in accordance with the Data Protection Act (1998). A unique trial identification number is used on all follow-up questionnaires. 


\section{Data collection and management}

Self-reported questionnaire data is scanned using FORMIC software. This is an automated data capture package which captures questionnaire text. In addition to internal FORMIC system validation checks, multiple external validation checks will be applied: at the point of receipt of case record form (CRF), on entry scanning to FORMIC, on transfer from FORMIC into internal database software and further checks for data outliers during statistical analysis.

\section{Health economic analysis}

Two economic evaluations will be undertaken- $a$ within trial evaluation will compare the incremental costs per quality adjusted life year (QALY) at follow-up and, second, a decision analytic cost-effectiveness model will be used to estimate the expected incremental cost per QALY. For both analyses, the perspective will be that of the UK NHS and social services.

The within trial analysis will use the EQ-5D. ${ }^{30} 31$ The costs of delivering MFFP are greater than exercise; therefore MFFP will have to prove more effective than exercise to be cost-effective, that is, superior. Costs and outcomes will be discounted at $3.5 \%$ per annum and probabilistic sensitivity analysis will be undertaken using the non-parametric bootstrap. The decision analytic costeffectiveness analysis model will use a lifetime time horizon to capture the full impact of any mortality differences on the long-term cost-effectiveness. The methods for estimating health-related quality of life and discounting will be the same as for the within trial analysis. Probabilistic sensitivity analyses will be undertaken using Monte Carlo simulation techniques.

\section{Trial monitoring}

A TSC and independent data monitoring committee (iDMC) will monitor the trial at least every 12 months. One of the aims of the pilot study was to investigate whether the trial would fit within a futility framework. After extensive exploration and consideration of the different aspects of statistical and practical futility, a decision was undertaken, with the iDMC and TSC to proceed to the main trial without interim analysis of fracture rates.

\section{Adverse event management}

A safety reporting protocol has been developed for related and unexpected serious adverse events (AEs) and directly attributable AEs. An AE is defined as any untoward medical occurrence in a subject which does not necessarily have a causal relationship with treatment. The trial chief investigator will determine whether AEs require reporting to the trial sponsor, iDMC and Ethics Committee, in accordance with the safety reporting protocol.

\section{Ethical issues}

The study protocol has approval from the National Research Ethics Service (REC reference 10/H0401/36; V.3.1 21/05/2013; see online supplementary material). Regional and site-specific approvals have been obtained from NHS Research and Development offices. A number of ethical issues were addressed in relation to the trial design. First, consent to cluster randomisation: general practices consent to randomisation, to approach potential participants for invitation and consent to provide the intervention as allocated. Second, individual consent is obtained for the intervention for recommended interventions as part of usual clinical care and follows best practice guidance on consent to treatment. Third, participation in research assessments and access to patient records: individual consent is obtained for access to medical records, for postal and telephone follow-up. We have involved relevant user groups and patient public representatives to inform the design and implementation of the trial. Approval was granted by the Derbyshire Research Ethics Committee.

The study team is committed to full disclosure of the results of the trial. We will adhere to defined authorship criteria as per the International Committee of Medical Journal Editors. The funder will take no role in the analysis or interpretation of results.

\section{DISCUSSION}

This will be the first ever large-scale multicentre clinical trial to record fracture as the primary outcome in a falls prevention study comparing advice only, versus MFFP and exercise in community-dwelling older adults. The cluster trial design where the clusters are assembled prior to randomisation provides methodological rigour and the ability to track uptake and effectiveness at different stages of the intervention. We have used several novel strategies to maximise the efficiency of the design, including control of the cluster size through random sampling, and the use of random subsampling to reduce the burden of follow-up for outcome measures that do not require as much statistical power for definitive analysis. The large sample size and multiple approaches to accurate capture of fractures and falls will allow considered analyses of primary outcomes and useful secondary analyses of moderator and mediator variables.

\section{Author affiliations}

${ }^{1}$ Warwick Clinical Trials Unit, Division of Health Sciences, University of Warwick, Coventry, UK

${ }^{2}$ Leeds Institute of Health Sciences, University of Leeds, Leeds, UK

${ }^{3}$ Royal Devon \& Exeter Hospital, Exeter, UK

${ }^{4}$ School of Health and Life Sciences, Glasgow Caledonian University, Glasgow, UK

${ }^{5}$ OIder Persons' Unit, Guys and St Thomas' NHS Foundation Trust, St Thomas' Hospital, London, UK

${ }^{6}$ Nuffield Department of Orthopaedics Rheumatology \& Musculoskeletal Sciences, Botnar Research Centre, University of Oxford, Oxford, UK 
Acknowledgements The authors are very grateful to the general practices, research teams, falls services and physiotherapy teams who are collaborating in this study. The authors also extend very grateful thanks to the study participants.

Collaborators PreFIT Study Group: Chief Investigator: Professor Sarah E Lamb. Co-investigators (Grant holders): Professor Martin Underwood, Professor Finbarr Martin, Professor Lucy Yardley, Professor Dawn Skelton, Professor Keith Willett, Professor Sandra Eldridge, Dr Anne-Marie Slowther, Dr Sarah Duggan. Trial Research Lead: Dr Julie Bruce. Senior Project Manager: Susie Hennings (SH). Trial Co-ordination/Administration: Emma Withers, Susie Hennings, Rhys Mant, Rishpal Rai, Craig Turner, Agata Andrews, Rachael Fearn. Research Fellows/Nurses: Susanne Finnegan, Nicola Walker, Rachel Potter. Trial statistician: Dr Ranjit Lall. Health Economists: Claire Hulme, Roberta Longo (RLo). Clinical Intervention Trainers: Susanne Finnegan, Dr Katherine Westacott, Dr Shvaita Ralhan, Dr Ray Sheridan, Dr Jonathan Treml. Regional Principal Investigators: Dr Ray Sheridan (Devon Region), Ms Jackie Riglin (Cambridge Region); Mr Harm Gordjin (Warwickshire Region); Dr Ruma Dutta (Worcestershire Region); Ms Jo Burns (Hereford Region), Dr Jonathan Treml (Birmingham \& Black Country Region), Dr Fiona Shaw, Dr John Davison (Newcastle Region). Data Programming team: Ade Willis, Chockalingam Muthiah, Henry Adjei.

Contributors SEL is the chief investigator who conceived the original study design and obtained funding. SEL, JB, RL, MU, EJW, CH and RLo participated in the design and conduct of the study. EJW coordinated study administration, acquisition of trial data and administrative support. SH provided senior project support. SEL, SF, JB and RS participated in the design of interventions; SF coordinates delivery of interventions. RLa is the senior trial statistician responsible for conduct of statistical analysis. RLo participated in the design of the health economics component and will undertake the health economics analysis under direction of $\mathrm{CH}$. JB drafted the manuscript. All authors critically revised the manuscript for important intellectual content and approved the final manuscript. This protocol is published on behalf of the PreFIT Study Group.

Funding The PreFIT study is funded by the National Institute of Health Research Technology Assessment Programme (NiHR HTA), project number $08 / 14 / 41$. The views expressed in this publication are those of the authors and not necessarily those of the NIHR or Department of Health. This project benefited from facilities funded by Birmingham Science City Translational Medicine Clinical Research and Infrastructure Trials Platform, with support from Advantage West Midlands (AWM). The trial sponsor is the University of Warwick. The trial started in September 2010 and is funded until 2018.

Competing interests DS is a Director of Later Life Training, a not for profit training company that qualifies Otago exercise programme leaders. The other authors declare they have no competing interests. DS joined the study team after the decision to adopt the Otago programme, and has had no role in training health professionals within the Pre-FIT trial.

\section{Ethics approval Derbyshire Research Ethics Committee.}

Provenance and peer review Not commissioned; peer reviewed for ethical and funding approval prior to submission.

Data sharing statement The trial statisticians/analysts and iDMC will have access to the data set for the analysis of trial outcomes. The Chief Investigator (Lamb) will have access to the data and will take full responsibility for the analysis and publication of the results. Once the main analyses have been undertaken, data will be available to principal and other investigators subject to approval of data analysis plans by the steering committee and compliance with the University of Warwick Standard Operating Procedures on Data Management and Sharing. We will comply with Data Sharing Policies that may be instituted by the Funder (National Institute of Health Research) during the lifetime of the project.

Open Access This is an Open Access article distributed in accordance with the Creative Commons Attribution Non Commercial (CC BY-NC 4.0) license, which permits others to distribute, remix, adapt, build upon this work noncommercially, and license their derivative works on different terms, provided the original work is properly cited and the use is non-commercial. See: http:// creativecommons.org/licenses/by-nc/4.0/

\section{REFERENCES}

1. Bischoff-Ferrari HA. The role of falls in fracture prediction. Curr Osteoporos Rep 2011;9:116-21.

2. Rubenstein LZ, Powers CM, MacLean CH. Quality indicators for the management and prevention of falls and mobility problems in vulnerable elders. Ann Intern Med 2001;135(8 Pt 2):686-93.

3. Association $\mathrm{BO}$. The care of patients with fragility fracture. London: British Orthopaedic Association, 2007.

4. Ftouh S, Morga A, Swift C, et al. Management of hip fracture in adults: summary of NICE guidance. BMJ 2011;342:d3304.

5. Deandrea S, Lucenteforte E, Bravi F, et al. Risk factors for falls in community-dwelling older people: a systematic review and meta-analysis. Epidemiology 2010;21:658-68.

6. NICE. Clinical practice guideline for the assessment and prevention of falls in older people. London: National Institute for Clinical Excellence, 2004.

7. NICE. Falls: the assessment and prevention of falls in older people. National Institute for Clinical Excellence (NICE), 2013 Contract No. CG161.

8. Lamb SE, Fisher JD, Gates S, et al. A national survey of services for the prevention and management of falls in the UK. BMC Health Serv Res 2008;8:233.

9. RCP. Older people's experiences of therapeutic exercise as part of a falls prevention service-patient and public involvement. London: RCP, 2012.

10. Gillespie LD, Robertson MC, Gillespie WJ, et al. Interventions for preventing falls in older people living in the community. Cochrane Database Syst Rev 2012;9:CD007146.

11. Gates S, Fisher J, Cooke M, et al. Multifactorial assessment and targeted intervention for preventing falls and injuries among older people in community and emergency care settings: systematic review and meta-analysis. BMJ 2008;336:130-3.

12. Campbell MK, Piaggio G, Elbourne DR, et al. Consort 2010 statement: extension to cluster randomised trials. BMJ 2012;345:e5661.

13. Lamb S, McCabe C, Becker C, et al. The optimal sequence and selection of screening test items to predict fall risk in older disabled women: the Women's Health and Aging Study. J Gerontol A Biol SCi Med Sci 2008:63:1082-8.

14. OEP. Otago exercise programme to prevent falls in older adults. In: Cambpell JA, Borke RC, eds. Otago medical school. Otago: University of Otago, 2003:1-69.

15. Campbell AJ, Robertson MC, Gardner MM, et al. Randomised controlled trial of a general practice programme of home based exercise to prevent falls in elderly women. BMJ 1997;315:1065-9.

16. Tinetti M, Baker D, McAvay G, et al. A multifactorial intervention to reduce the risk of falling among elderly people living in the community. N Engl J Med 1994;331:821-7.

17. AGS/BGS. Guideline for the prevention of falls in older persons. American Geriatrics Society, British Geriatrics Society, and American Academy of Orthopaedic Surgeons Panel on Falls Prevention. J Am Geriatr Soc 2001;49:664-72.

18. Hoffman TC, Glasziou PP, Boutron I, et al. Better reporting of interventions: template for intervention description and replication (TIDieR) checklist and guide. BMJ 2014;348:g1687.

19. Lamb S, Jørstad-Stein E, Hauer K, et al. Group POC. Development of a common outcome data set for fall injury prevention trials: the Prevention of Falls Network Europe consensus. J Am Geriatr Soc 2005;53:1618-22.

20. Hauer K, Lamb SE, Jorstad EC, et al. PROFANE-Group. Systematic review of definitions and methods of measuring falls in randomised controlled fall prevention trials. Age Ageing 2006;35:5-10.

21. Strawbridge W, Shema S, Balfour J, et al. Antecedents of frailty over three decades in an older cohort. J Gerontol B Psychol Sci Soc Sci 1998;53:S9-16.

22. Shua-Haim J, Koppuzha G, Gross J. A simple scoring system for clock drawing in patients with Alzheimer's disease. J Am Geriatr Soc 1996; $44: 335$.

23. Ware JE. The SF-12: An even shorter health survey:Version 2.0. 2002.

24. Brooks R. EuroQol: the current state of play. Health Policy 1996;37:53-72.

25. Donaldson L, Reckless I, Scholes S, et al. The epidemiology of fractures in England. J Epidemiol Community Health 2008;62:174-80. 
26. Office for National Statistics, 2011 Census: Aggregate data (England and Wales). UK Data Census Support. Downloaded from: http: infuse.ukdataservice.ac.uk.

27. Eldridge S, Spencer A, Cryer C, et al. Why modelling a complex intervention is an important precursor to trial design: lessons from studying an intervention to reduce falls-related injuries in older people. J Health Serv Res Policy 2005;10: 133-42.

28. Pincus T, Miles C, Froud R, et al. Methodological criteria for the assessment of moderators in systematic reviews of randomised controlled trials: a consensus study. BMC Med Res Methodol 2011;11:14.

29. Brookes $\mathrm{S}$, Whitley E, Peters $\mathrm{T}$, et al. Subgroup analyses in randomised controlled trials: quantifying the risks of false-positives and false-negatives. Health Technol Assess 2001;5:1-56.

30. Dolan P. Modeling valuations for EuroQol health states. Med Care 1997;35:1095-108.

31. Kind P, Dolan P, Gudex C, et al. Variations in population health status: results from a United Kingdom national questionnaire survey. BMJ 1998;316:736-41. 